\title{
Abordagem fonoaudiológica em pacientes com fissura labiopalatal em serviço especializado de alta complexidade na região oeste do Paraná
}

Speech therapy approach in patients with labiopalatal cleft in high complexity specialized service in the west region of Paraná

Abordaje de la terapia del linguaje pacientes com fissura labiopalatal em servicio especializado de alta complejidad em la región oeste de Paraná

Recebido: 04/08/2021 | Revisado: 13/08/2021 | Aceito: 18/08/2021 | Publicado: 20/08/2021

Celina Cabral

ORCID: https://orcid.org/0000-0002-9148-0227

Universidade Estadual do Oeste do Paraná, Brasil E-mail: celinacabrall@gmail.com

Maria Grazielle Paiva Barreto Santana Lopes ORCID: https://orcid.org/0000-0001-8782-3058 Universidade Estadual do Oeste do Paraná, Brasil E-mail: grazzi_pl@outlook.com

Daniela Lopes de Oliveira ORCID: https://orcid.org/0000-0002-8783-0976 Universidade Estadual do Oeste do Paraná, Brasil E-mail: danilopesbr@yahoo.com.br

Mariângela Monteiro de Melo Baltazar ORCID: https://orcid.org/0000-0001-6395-9516 Universidade Estadual do Oeste do Paraná, Brasil E-mail: mmmwgb@uol.com.br

\begin{abstract}
Resumo
Introdução: as fissuras labiopalatinas são as malformações craniofaciais mais frequentemente identificadas em recémnascidos vivos. De maneira geral, o tratamento das fissuras objetiva corrigi-las cirurgicamente, bem como corrigir os problemas associados, como alterações nas funções da deglutição, mastigação, sucção, audição, fala entre outras consequências que possam surgir. Objetivo: discorrer sobre a atuação e o papel do fonoaudiólogo em equipe multidisciplinar de um centro de reabilitação em anomalias craniofaciais, com enfoque na fissura labiopalatina. Metodologia: trata-se de um relato de experiência produzido a partir das vivências profissionais da equipe de fonoaudiologia em um Centro de atenção e Pesquisa em Anomalias Craniofaciais. Resultados e discussão: a atuação fonoaudiológica no tratamento especializado das fissuras labiopalatinas é complexa e processual, sendo realizado um acompanhamento longo, no qual os resultados são uma combinação de fatores, como o envolvimento e assiduidade familiar no tratamento, colaboração do paciente, cirurgias e a atuação de cada profissional da equipe. $O$ resultado final do tratamento deve refletir na qualidade de vida e desenvolvimento geral do paciente. Conclusão: entende-se como fundamental a participação do fonoaudiólogo neste processo, com atuação ativa desde o início do tratamento, visando contribuir no desenvolvimento das habilidades comunicativas em geral, bem como detectar possíveis alterações e intervir precocemente.
\end{abstract}

Palavras-chave: Fissura labial; Fissura palatina; Fonoterapia; Desenvolvimento infantil.

\begin{abstract}
Introduction: cleft lip and palate are the most frequently identified craniofacial malformations in live newborns. In general, the treatment of fissures aims to correct them surgically, as well as to correct the associated problems, such as alterations in the functions of swallowing, chewing, sucking, hearing, speaking, among other consequences that may arise. Objective: do discuss the role of the speech therapist in a multidisciplinary team of a rehabilitation center for craniofacial anomalies, focusing on cleft lip and palate. Methodology: this is an experience report produced from the professional experiences of the speech therapy team in a Care and Research Center for Craniofacial Anomalies. Results and discussion: the speech therapy role in the specialized treatment of cleft lip and palate is complex and procedural, with a long follow-up being carried out, in which the results are a combination of factors, such as family involvement and attendance in the treatment, patient collaboration, surgeries and performance of each professional on the team. The final result of the treatment must reflect the quality of life and general development of the patient. Conclusion: it is understood as fundamental the participation of the speech therapist in this process, with active role since the beginning of the treatment, aiming to contribute to the development of communication skills in general, as well as detect possible changes and intervene early.
\end{abstract}

Keywords: Cleft lip; Cleft palate; Speech therapy; Child development. 


\begin{abstract}
Resumen
Introducción: el labio y paladar hendido son las malformaciones craneofaciales identificadas con mayor frecuencia en los recién nacidos vivos. En general, el tratamiento de fisuras tiene como objetivo corregirlas quirúrgicamente, así como corregir los problemas asociados, como alteraciones en las funciones de tragar, masticar, succionar, oír, hablar, entre otras consecuencias que puedan surgir. Objetivo: discutir el rol y el rol del logopeda en un equipo multidisciplinario de un centro de rehabilitación de anomalías craneofaciales, con foco en labio leporino y paladar hendido. Metodología: se trata de un relato de experiencia elaborado a partir de las experiencias profesionales del equipo de logopedia en un Centro de Atención e Investigación de Anomalías Craneofaciales. Resultados y discusión: el papel de la logopedia en el tratamiento especializado de el labio y paladar hendido es complejo y procedimental, con un seguimiento prolongado, en el que los resultados son una combinación de factores, como la implicación familiar y la asistencia al tratamiento, el paciente, colaboración, cirugías y desempeño de cada profesional del equipo. El resultado final del tratamiento debe reflejar la calidad de vida y el desarrollo general del paciente. Conclusión: se entiende como fundamental la participación del logopeda en este proceso, con rol activo desde el inicio del tratamiento, con el objetivo de contribuir al desarrollo de las habilidades comunicativas en general, así como detectar posibles cambios e intervenir precozmente.
\end{abstract}

Palabras clave: Labio leporino; Paladar Hendido; Terapia del languaje; Desarollo Infantil.

\title{
1. Introdução
}

As fissuras labiopalatinas (FLP) são as malformações craniofaciais mais frequentemente identificadas em recémnascidos vivos (Martelli et al., 2012). Constituem malformações decorrentes de falhas na fusão dos processos faciais, que ocorrem entre a $4^{\circ}$ e $12^{\circ}$ semanas de idade gestacional, período de maior sensibilidade para o desenvolvimento da face e cavidade oral (Borges et al., 2014; Freitas et al., 2011).

A classificação proposta por SPINA em 1979, utiliza o forame incisivo como ponto de referência é a mais utilizada no Brasil, por ser simples e objetiva. Considerando este ponto de referência, as fissuras são classificadas de acordo com o modo que atingem o forame incisivo e as estruturas anatômicas adjacentes: Fissura pré-forame acomete lábio e rebordo alveolar; fissura transforame: acomete lábio, rebordo alveolar, palato duro, palato mole e úvula; fissura pós forame: palato duro e/ou palato mole, podendo ainda ser completa e incompleta, conforme a proximidade com o forame; e ainda fissura rara de face que atinge outras estruturas da face. Também são definidas conforme a extensão, sendo completas ou incompletas de forma unilateral, bilateral ou mediana (Borges et al., 2014; Lorenzzoni, Carcereri, \& Lucks., 2014).

As FLP podem se apresentar como parte de um quadro sindrômico, estando associadas a outras deformidades ou como fissuras isoladas, sendo estas não sindrômicas, sem demais deformidades físicas e de desenvolvimento (Freitas et al., 2011). Além disso, são apontados como etiologias das fissuras, fatores genéticos tais como polimorfismos e mutações que interagem com os fatores ambientais: déficit nutricional, uso de drogas, etilismo, tabagismo e exposição a radiação (SouzaFreitas, Dalben, \& Freitas, 2014).

Durante a gestação, os pais sentem-se ansiosos para o nascimento do bebê, o que causa grandes expectativas e emoções aliadas à ansiedade por ver o rosto da criança. Entretanto, em casos de FLP, os pais são surpreendidos, o que por vezes leva a conflitos emocionais, tristeza ou até mesmo sentimento de negação, devido a não concretização daquela idealização do filho. Esses sentimentos podem gerar um rompimento de parte dos planos e anseios, bem como a culpabilização pelos pais sobre a impossibilidade de gerar o "filho perfeito". Inicialmente, os pais devem ser orientados a procurar tratamento em centros especializados no tratamento de FLP, acompanhamento este que refletirá de forma eficaz na reabilitação da criança (Alves, Carrara, \& Costa, 2005; Brasil et al., 2007).

De maneira geral, o tratamento das fissuras objetiva primeiramente corrigi-las cirurgicamente, além disso, é necessário tratar os problemas associados como alterações nas funções da deglutição, mastigação, sucção, audição, fala, oclusão entre outras consequências que possam surgir. Sendo assim, o tratamento deve ser realizado por uma equipe multidisciplinar, pois somente desta forma é possível contemplar todas as alterações e particularidades de acordo com a complexidade que cada caso impõe, desde as demandas estéticas até as funcionais (Di-Bernardo et al., 2017). 
O resultado de todas as intervenções realizadas pela equipe a longo prazo, deve ser a reabilitação integral do paciente, compreendendo desde a correção cirúrgica da anomalia até a reabilitação da fala e demais funções/estruturas acometidas. $\mathrm{O}$ tratamento, quando adequado, reflete inclusive na inserção social e qualidade de vida dos pacientes (Almeida et al., 2017).

A equipe fonoaudiológica em um centro de referência em reabilitação multidisciplinar desenvolve as funções de avaliação, (re)habilitação e prevenção de alterações no sistema estomatognático e suas funções desde que sua etiologia esteja relacionada a anomalias craniofaciais. $\mathrm{O}$ objetivo do presente relato é discorrer sobre a atuação do fonoaudiólogo inserido em um centro de reabilitação em anomalias craniofaciais, com enfoque na FLP.

\section{Metodologia}

Trata-se de um relato de experiência de caráter qualitativo, produzido a partir das vivências profissionais da equipe de fonoaudiologia no Centro de atenção e Pesquisa em Anomalias Craniofaciais (CEAPAC). O CEAPAC é um anexo ao Hospital Universitário do Oeste do Paraná (HUOP), que iniciou suas atividades em 2013 oferecendo tratamento especializado de alta complexidade para os pacientes com fissuras labiopalatais. Atende pacientes de cidades das regiões: Oeste, Sudoeste e Noroeste do Paraná (PR) (Matos et al., 2019). É composto por uma equipe multiprofissional de diversas áreas: fonoaudiologia, enfermagem, geneticista, odontologia e especialidades como ortodontia, odontopediatria, cirurgia bucomaxilofacial, periodontia, implantodontia e prótese, medicina e especialidades como cirurgia plástica, cirurgia pediátrica, pediatria, otorrinolaringologia, neurocirurgia pediátrica, anestesiologia, além de nutrição, fisioterapia, psicologia e serviço social.

Em 2018, obteve a habilitação em alta complexidade em lesões lábio palatais pelo Ministério da saúde e desde março de 2020, o CEAPAC passou a contar com o Programa de Residência Multiprofissional em Reabilitação Integral das Anomalias Craniofaciais, contemplando as áreas de fonoaudiologia, fisioterapia, psicologia, odontologia, serviço social, nutrição, genética e enfermagem, o que proporcionou um crescimento na equipe que compõe o serviço, associando o ensino, a pesquisa e a extensão, configurando-se numa oportunidade de capacitação dos profissionais na área de reabilitação das fissuras.

De caráter ambulatorial, os atendimentos ocorrem de segunda a sexta-feira sendo agendados previamente via sistema GSUS a partir da atenção básica da macrorregião.

\section{Resultados e Discussão}

Um dos pilares fundamentais no tratamento da FLP é a equipe multidisciplinar, sendo imprescindível a discussão dos casos entre a equipe, que deve ser composta por: fonoaudiólogo, fisioterapeuta, psicólogo, nutricionista, ortodontista, cirurgião bucomaxilofacial, enfermeiro, assistente social, cirurgião plástico, odontopediatra, pediatra, otorrinolaringologista, geneticista, dentre outras especialidades (Pereira, 2019; Signor, 2019). A intervenção precoce é fundamental pois prestará a assistência necessária na garantia da qualidade de vida da criança, refletindo em melhores resultados estéticos e funcionais evitando-se assim, as sequelas (Zhou et al., 2016; Costa, et al., 2018).

No setor de fonoaudiologia é ofertado o acompanhamento desde os primeiros dias de vida da criança, porém, não há uma faixa etária específica para o atendimento. O público do centro compreende desde gestantes com diagnóstico confirmado de fissura, bebês, adultos e até idosos. Inicialmente, parte-se de uma entrevista inicial com o acolhimento da demanda familiar permeado por uma escuta qualificada para posteriores orientações iniciais. A avaliação é feita por meio de protocolos específicos, para a compreensão das características da malformação, bem como impacto nas funções do sistema estomatognático: sucção, respiração, deglutição, mastigação e fala. Quando necessário é realizada a avaliação de linguagem oral, compreensão e expressão. Além disso, também aplica-se a avaliação auditiva, por meio dos exames de audiometria tonal limiar, logoaudiometria, imitanciometria e Potencial Evocado Auditivo de Tronco Encefálico-PEATE. Sempre que identificadas alterações auditivas, os pacientes são encaminhados para o tratamento adequado. A atuação estende-se também 
nas demais etapas de desenvolvimento infantil, adolescência e idade adulta sempre que necessário. Quando o tratamento multiprofissional não for realizado precocemente, maiores serão as chances de ocorrerem alterações que necessitam de intervenção na idade adulta (Signor, 2019).

Cabe ao fonoaudiólogo, que trabalha na reabilitação de pacientes com FLP, ater-se aos seguintes fatores: desenvolvimento dos aspectos oromiofuncionais, linguagem oral, escrita e aprendizagem, fala, voz e audição (Costa et al., 2018). A intervenção começa ainda no período gestacional, quando for diagnosticado nesta fase com orientações aos pais. A partir disso, o profissional atuará com o recém-nascido, inicialmente, quando a criança apresenta dificuldades no aleitamento materno é necessário o uso de mamadeiras bem como a adaptação do bico mais adequado para a criança, que pode ser ortodôntico, de látex, bicos convencionais, bico dosador. Independentemente do tipo, indica-se aquele que a criança apresentar melhor adaptação, pois o foco inicial é voltado em manter a saúde geral bem como estado nutricional do bebê (Almeida et al., 2018; Santos, Janini, \& Oliveira 2019).

De acordo com Altmann e colaboradores (2005), no bebê com fissura palatina ocorre alteração no posicionamento da língua, mantendo-se posteriorizada. Sendo assim, ocorre grande estimulação do dorso lingual, podendo implicar na ocorrência de DACs durante o desenvolvimento da fala. Diante disso, o tratamento fonoaudiológico precoce objetiva: não produzir sons de pressão oral até a palatoplastia; orientar os responsáveis para, de forma lúdica, realizar a breve oclusão das narinas enquanto o bebê vocaliza, isso favorecerá a percepção da pressão intraoral; promover estímulos sensoriais na região anterior da boca, evitando a ocorrência dos DACs e favorecendo o desenvolvimento da fala. Também pode-se utilizar o modelador nasoalveolar (NAM), um aparelho ortopédico que se propõe a minimizar a deformidade inicial quando utilizado precocemente. Tal dispositivo promove o alinhamento nos tecidos antes da correção cirúrgica do lábio e nariz podendo levar a melhores resultados cirúrgicos (Lee, Grayson, \& Cutting, 2004).

Todas as etapas do tratamento são permeadas por orientações aos responsáveis, desta forma, destaca-se a importância do estabelecimento de um vínculo entre a família e o profissional, sendo este um aspecto essencial, independente da especialidade. No âmbito fonoaudiológico, orienta-se quanto aos cuidados sobre a amamentação, tipos de utensílios (quando não for amamentação ao seio), postura verticalizada durante a alimentação, desenvolvimento da fala, linguagem oral e audição. Destaca-se aqui as orientações pré-cirúrgicas, que são realizadas antes da queiloplastia (cirurgia para a correção do lábio) e palatoplastia (cirurgia para a correção do palato), pois, são procedimentos que exigem cuidados específicos no período póscirúrgico.

Os responsáveis pela criança são orientados quanto a forma de oferta da alimentação, bem como o que deve ser evitado, visando favorecer sua recuperação para que não ocorra deiscência, infecção, ou qualquer outra intercorrência na ferida cirúrgica. As orientações principais englobam: não sugar, para tanto recomenda-se o uso da mamadeira com bico dosador (colher) e/ou copo convencional (sem canudos); não introduzir objetos na cavidade oral como: brinquedos, chupeta ou o próprio dedo; evitar o choro exagerado. Os cuidados iniciais no pós-operatório implicam inclusive, no resultado final da cirurgia. Ressalta-se que para a queiloplastia, nos casos em que o bebê está mamando no peito, deve-se manter o aleitamento materno, não havendo restrições. Entretanto, na palatoplastia isso não é permitido, devido ao risco de causar a deiscência dos pontos.

Após 30 dias de pós operatório de queiloplastia e a liberação do cirurgião plástico, tem-se início as orientações referentes à exercícios funcionais labiais, os quais envolvem a mobilidade e tonicidade da musculatura orbicular dos lábios, de maneira lúdica. Conjuntamente, combina-se com as massagens na região da cicatriz. Esta é uma intervenção realizada pelo setor de fonoaudiologia, onde, além de aplicar as técnicas adequadas é realizada a orientação e prática com o responsável pela criança, para que as massagens também sejam realizadas em casa. Lembrando que a direção do movimento e pressão 
adequados são fundamentais, devendo ser realizadas todos os dias, por um período de seis meses de pós operatório, para atingir os resultados esperados.

As massagens são realizadas na cicatriz, com movimentos retilíneos e circulares, de cima para baixo, por meio de toques, pressões, alisamento e trações. Devem ser realizadas três vezes ao dia. O objetivo de massagear a região da cicatriz é promover aquecimento, soltura, vascularização muscular e tecidual favorecendo o processo de cicatrização e funcionalidade (Rossi et al., 2005). Com esta prática, tem-se observado excelentes resultados estéticos e funcionais, com nítida diferença entre aqueles casos que realizam as massagens diariamente e aqueles que não o fazem ou fazem com menor frequência. Inclusive, destaca-se como um percalço nesta etapa do tratamento a dificuldade que muitos cuidadores referem na hora de realizar as massagens em casa, tendo em vista que a criança chora e manifesta desconforto, muitos acabam deixando de fazer diariamente, implicando negativamente no resultado da cirurgia.

Após 30 dias da palatoplastia, quando não ocorrem intercorrências como a deiscência dos pontos, dá-se continuidade ao acompanhamento fonoaudiológico. São realizadas então, orientações sobre o desenvolvimento da fala e linguagem, de forma a prevenir a instalação de distúrbios articulatórios compensatórios (DACs), comuns nos casos de FLP. Além disso, são orientados exercícios que promovam a estimulação do palato mole visando a sua funcionalidade de modo adequado, direcionamento do fluxo aéreo para a cavidade oral, treino de sucção, sons e jogos vocálicos. Neste processo, ao detectar qualquer alteração estrutural que possa estar afetando a funcionalidade do palato mole o paciente é reavaliado pela equipe de cirurgia, visto que limitações anatômicas na região do esfíncter velofaríngeo, como palato curto, dificultam a evolução no tratamento fonoaudiológico, podendo ter por desfecho uma disfunção velofaríngea (DVF), com grandes prejuízos ao paciente. Afirma-se portanto, que bons resultados no desenvolvimento da fala também dependem de um bom resultado cirúrgico (Hosseinbad et al, 2015; Shaffer et al., 2020) e a interlocução da equipe cirúrgica com a fonoaudiologia é fundamental para o sucesso da abordagem integral, necessária ao paciente.

Dentre os aspectos relevantes para uma adequada reabilitação após a palatoplastia, enfatiza-se a reparação correta do palato, pois é o que irá determinar se a fonoterapia vai obter bons resultados no desenvolvimento da fala, que é afetada por alterações no mecanismo velofaríngeo (Hosseinbad et al., 2015). Desta forma, quando identificada a necessidade no acompanhamento após a cirurgia, o paciente é encaminhado para uma reavaliação com o cirurgião plástico, o qual avaliará a necessidade de uma nova intervenção cirúrgica para correção visando favorecer a funcionalidade do palato (Signor, 2019).

Sempre que são identificados os casos que demandam terapia fonoaudiológica semanal, são realizados os encaminhamentos junto a equipe de serviço social, para que a criança seja atendida na cidade de origem, em serviço de atenção primária ou secundária a saúde. A equipe de fonoaudiologia subsidia os municípios com orientações e prescrições de exercícios específicos, buscando, sempre que possível, contato direto com as fonoaudiólogas da atenção básica. Os retornos para reavaliações são mensais e deste modo, com ações descentralizadas para os níveis de menor complexidade, possibilita-se a integralidade da atenção ao portador de fissura.

Evidencia-se a necessidade de uma integração real entre a equipe multidisciplinar, dado que são diferentes olhares e intervenções em prol de um objetivo comum: a reabilitação integral do paciente com FLP.

Em síntese, a atuação fonoaudiológica no tratamento especializado das FLP é complexa e processual, sendo que é realizado um acompanhamento considerado longo, no qual os resultados são uma combinação de fatores, como o envolvimento e assiduidade familiar no tratamento, colaboração do paciente, cirurgias e a atuação de cada profissional da equipe. $\mathrm{O}$ resultado final do tratamento deve refletir na qualidade de vida e desenvolvimento geral do paciente. 


\section{Conclusão}

Destaca-se que existem diferenças nos protocolos de manejo da FLP nos diferentes centros especializados espalhados pelo Brasil, o presente trabalho teve como objetivo discorrer sobre a atuação fonoaudiológica em uma equipe especializada.

Conclui-se que a participação do fonoaudiólogo no processo de reabilitação da FLP é fundamental, com participação ativa desde o início do tratamento, visando contribuir no desenvolvimento das habilidades comunicativas em geral, bem como, detectar possíveis alterações para intervir o mais precocemente possível.

A equipe como um todo deve ter como objetivo principal do tratamento a minimização das alterações impostas pela anomalia. Cada etapa do tratamento exigirá uma cronologia adequada para as intervenções, sempre permeado pelo princípio da interdisciplinaridade, visando promover melhorias na qualidade de vida, desenvolvimento global e inserção social do paciente.

\section{Referências}

Almeida, A. M. F. L., Chaves, S. C. L., Santos, C. M. L., \& Santana S. F. (2017). Atenção à pessoa com fissura labiopalatina: proposta de modelização para avaliação de centros especializados no Brasil. Saúde Debate.41, 156-166. https://bce.unb.br/wp-content/uploads/2021/05/Estilo-APA_05-2021.pdf

Altmann, E., Vaz, A. C, Paula, M. B., \& Khoury, R. B. (2005). Tratamento precoce. In: Altmann, E. organizador. Fissuras labiopalatinas. Barueri: Pró-Fono. 291-324.

Alves, A., Carrara, C. F. C., \& Costa, B. (2005). Influência da conduta dos pais sobre o comportamento de crianças com fissura de lábio e/ou palato diante do tratamento odontológico realizado no Hospital de Reabilitação de Anomalias Craniofaciais. Pediatr Mod. 44(3), 321-326. https://repositorio.usp.br/item/001547288

Borges, A. R., Mariano, L., Sá, J., Medrado, A. P., Veiga, P. C., \& Reis, S. R. A. (2014). Fissuras labiais e/ou palatinas não sindrômicas: determinantes ambientais e genéticos. Revista Bahiana de Odontologia. 5(1), 48-58. https://www5.bahiana.edu.br/index.php/odontologia/article/download/329/266

Brasil, F. R., Tavano, L. A., Caramashi, S., \& Rodrigues, O. M. P. S. (2007). Escolha de parceiros afetivos: influência das sequelas de fissura labiopalatal. Paideia. 17(38), 375-387. https://www.scielo.br/j/paideia/a/h67YbqnjtWMtVYW9tqWfwBr/abstract/?lang=pt

Costa, V. C. R., Silva, R. C., Oliveira, I. F., Paz, L. B., Pogue, R., \& Gazzoni, L. (2018). Aspectos etiológicos e clínicos das fissuras labiopalatinas. Revista de Medicina e Saúde de Brasília. 7(2), 258-268. https://edisciplinas.usp.br/pluginfile.php/5588447/mod_resource/content/1/Costa\%20et\% 20al.\%2C\%202018\%20-\%20Aspectos\%20etiol\% C3\%B3gicos \%20e\%20c1\%C3\%ADnicos\%20das\%20fissuras\%20labiopalatinas.pdf

Di-Bernardo, B., Bellato, A., Moreira, M. A., Rodrigues, V. T, \& Pinto, C. (2017). Fissuras Lábio-Palatinas: Tipos de Tratamento- Revisão de Literatura. Revista de Divulgação Cientifica da ULBRA Torres. 13(3), 1-29. http://www.periodicos.ulbra.br/index.php/ci/article/view/3984

Freitas, J. A. S., Neves, L. T., Almeida, A. L. P. F., Garib, D. G., Trindade-Suedam, I. K., Uaedú, R. Y .F. ...\& Pinto, J. H. N. (2011). Rehabilitative treatment of cleft lip and palate: experience of the Hospital for Rehabilitation of Craniofacial Anomalies/USP (HRAC/USP) - Part 1: overall aspects. J Appl Oral Sci. 20(1), 9-15. https://www.scielo.br/j/jaos/a/nMj5X7bT8dgGpZJGFNFZ3Hm/?lang=en\&format=pdf

Hosseinabad, H. H., Derakhshandeh, F., Mostaajeran, F., Abdali, H., Davari, H. A., Hassanzadeh A. ... \& Kummer, A. W. (2015). Incidence of velopharyngeal insufficiency and oronasal fistulae after cleft palate repair: A retrospective study of children referred to Isfahan Cleft Care Team between 2005 and 2009. Int J Pediatr Otorhinolaryngol. 79(10), 1722-1726. https://pubmed.ncbi.nlm.nih.gov/26298624/

Lorenzzoni, D., Carcereri, D. L., \& Lucks, A. (2010). A importância do atendimento multiprofissional e interdisciplinar na reabilitação e promoção de saúde ao portador de fissura labiopalatal. Rev Odonto Cienc. 25(2), 198-203. http://revodonto.bvsalud.org/pdf/roc/v25n2/18.pdf

Lee, C., Grayson, B. H., \& Cutting, C. B. (2004). Prepubertal midface growth in unilateral cleft lip and palate following alveolar molding and gingivoperiosteoplasty. Cleft Palate Craniofac J. 41(4), 375-380. https://pubmed.ncbi.nlm.nih.gov/15297999/

Martelli, D. B. R., Machado, R. A., Swerts, M. S. O., Rodrigues, L. A. M., Aquino, S. N., \& Júnior, H. M. (2012). Non sindromic cleft lip and palate: relationship between sex and clinical extension. Braz J Otorhinolaryngol. 78(5), 116-120. http://www.bjorl.org.br/en-non-sindromic-cleft-lip-palate-articuloS1808869415303992

Matos, F. G. O. A., Santos, K. J. J., Baltazar, M. M. M., Fernandes, C. A. M., Marques, A. F. J., \& Luz, M. S. (2020). Perfil epidemiológico das fissuras labiopalatais de crianças atendidas em um centro de referência paranaense. REUFSM. 10(28), 1-14. https://periodicos.ufsm.br/reufsm/article/view/38654/html

Pereira, B. G. (2019). A multidisciplinaridade em fissuras labiopalatinas. Rev. Cient. Multidisc. UNIFLU. 4(2), $207-225$. http://www.revistas.uniflu.edu.br:8088/seer/ojs-3.0.2/index.php/multidisciplinar/article/view/213/135

Rossi, D. C., Di Ninno, Q. M. S., Silva, C. S., Rossi, K., \& Rodrigues, A. M. (2005). The effect of local mass on process of lip scar in children with unilateral cleft lip and palate surgery. Revista CEFAC. 7(2), 204-214. https://www.redalyc.org/pdf/1693/169320502008.pdf

Santos, S. R., Janini P. J., \& Oliveira S. M. H. (2019). A transição na amamentação de crianças com fenda labial e palatina. Rev. Esc. Anna Nery. $23(1)$, 1-7. https://www.scielo.br/j/ean/a/zTDqLcH3j6hHHkvJ7wPVgch/abstract/?lang=pt 
Research, Society and Development, v. 10, n. 10, e144101019062, 2021

(CC BY 4.0) | ISSN 2525-3409 | DOI: http://dx.doi.org/10.33448/rsd-v10i10.19062

Shaffer, A. D., Ford, M. D., Losee, J. E., Goldstein, J., Costello B. J., Grunwaldt, L. J., \& Jabbour, N. (2020). The Association Between Age at Palatoplasty and Speech and Language Outcomes in Children With Cleft Palate: An Observational Chart Review Study. The Cleft Palate-Craniofacial Journal. 57(2), 148160. https://pubmed.ncbi.nlm.nih.gov/31648546/

Signor, R. C .F. (2019). Abordagem fonoaudiológica nas fissuras orofaciais não sindrômicas: revisão de literatura. Rev Ciênc Med. 28(1):49-67. https://docs.bvsalud.org/biblioref/2020/01/1047805/med-5-00_4379.pdf

Souza- Freitas, J. Á., Dalben, G. S., \& Freitas, P. Z. (2004). Tendência familial das Fissuras labiopalatinas. R Dental Press Ortodon. Ortop. Facial.. 9(4), $74-$ 78. https://www.scielo.br/j/dpress/a/MjgSXdQSgk37dVCDyktytxN/?lang=pt

Zhou, Meng; Liu, Zong-xiang; Wang, Peng-lai \& Liu, Chao. (2016). Quality of life and its influential factors of children and adolescents with congenital cleft lip and palate. Shanghai kou qiang yi xue = Shanghai journal of stomatology. 25, 63-67. https://pubmed.ncbi.nlm.nih.gov/27063311/ 